\title{
Ion Cyclotron Wall Conditioning Experiments on Tore Supra in Presence of the Toroidal Magnetic Field
}

\author{
T. Wauters ${ }^{\text {a }}$, D. Douai ${ }^{\text {a }}$, S. Brémond ${ }^{\text {a }}$, E. de la Cal ${ }^{\text {b }}$, G. Lombard $^{\text {a }}$, A. \\ Lyssoivan $^{\mathrm{c}}$, B. Pegourié ${ }^{\mathrm{a}}$, E. Tsitrone ${ }^{\mathrm{a}}, \mathrm{M}$. Van Schoor ${ }^{\mathrm{c}}$ and \\ G. Van Oost ${ }^{\mathrm{d}}$ \\ ${ }^{a}$ CEA, IRFM, 13108 Saint-Paul-lez-Durance, France \\ ${ }^{b}$ Laboratorio Nacional de Fusión - CIEMAT, 28040 Madrid, Spain \\ ${ }^{c}$ ERM/KMS, LPP, 1000 Brussels, Belgium \\ ${ }^{d}$ Ghent University, Department of Applied Physics, 9000 Ghent, Belgium
}

\begin{abstract}
Wall conditioning techniques applicable in the presence of the high toroidal magnetic field will be required for the operation of ITER for tritium removal, isotopic ratio control and recovery to normal operation after disruptions. Recently ion cyclotron wall conditioning (ICWC) experiments have been carried out on Tore Supra in order to assess the efficiency of this technique in ITER relevant conditions. The ICRF discharges were operated in $\mathrm{He} / \mathrm{H}_{2}$ mixtures at the Tore Supra nominal field $(3.8 \mathrm{~T})$ and a RF frequency of $48 \mathrm{MHz}$, i.e. within the ITER operational space. RF pulses of $60 \mathrm{~s}$ (max.) were applied using a standard Tore Supra two-strap resonant double loop antenna in ICWC mode, operated either in $\pi$ or 0-phasing with a noticeable improvement of the RF coupling in the latter case. In order to assess the efficiency of the technique for the control of isotopic ratio the wall was first preloaded using a $\mathrm{D}_{2}$ glow discharge. After 15 minutes of ICWC in $\mathrm{He} / \mathrm{H}_{2}$ gas mixtures the isotopic ratio was altered from $4 \%$ to 50 $\%$ at the price of an important $\mathrm{H}$ implantation into the walls. An overall analysis comparing plasma production and the conditioning efficiency as a function of discharge parameters is given.
\end{abstract}

Keywords: Wall conditioning, ICRF discharge, Particle balance.

PACS: 52.25.Jm, 52.40.Hf, 52.50.Qt

\section{INTRODUCTION}

Recently the qualification of inter shot wall conditioning discharges became a high priority task for ITER [1] in order to mitigate tritium inventory build-up, to achieve the required low recycling levels at plasma startup and to reduce impurity levels after disruptions as well as after vacuum vents. The presence of a permanent high magnetic field in superconducting fusion machines, i.e. ITER, prevents the use of standard Glow Discharge Conditioning (GDC) during the active operational phase (the number of cycles of the ITER toroidal field coils is limited to 1000 for their whole lifetime [2]). RF plasmas are fully compatible with the presence of a high toroidal magnetic field. Their production mechanism is based on absorption of RF power in ion or electron cyclotron range of frequencies. Ion Cyclotron Wall Conditioning (ICWC) is presently considered as the most promising RF-technique for conditioning because of 
better discharge homogeneity (weakly dependent on $\mathrm{B}_{\mathrm{T}}$ ) and the ability to generate fast particles due to ion cyclotron absorption mechanisms [3]. To assess the efficiency of ICWC, dedicated experimental sessions were carried out on Tore Supra consisting of a parametric study concerning plasma production and preliminary optimization of the discharges, an assessment of the technique for controlling the wall isotopic ratio and the recovery to normal operation after disruptions. In the following a selection of experimental results is presented.

\section{EXPERIMENTAL SETUP}

The ICRF discharges were carried out at the Tore Supra nominal field (3.8 T) in a $\mathrm{He} / \mathrm{H}_{2}$ target gas mixture at a pressure of $\leq 0.1 \mathrm{~Pa}$ and a RF-frequency of $48 \mathrm{MHz}$, i.e. within the range of ITER frequency/field. In this way the fundamental ion cyclotron resonance layer for $\mathrm{H}$ was located on the low field side, approximately $25 \mathrm{~cm}$ away from the ICRF antenna. The RF power was delivered through a standard Tore Supra two-strap resonant double loop antenna with a range of tested powers from 30 to 200 $\mathrm{kW}$ (at generator side) applied in $\pi$ or 0-phasing, either in pulsed or continuous operation $(\max 60 \mathrm{~s}$ ). Injection of gas was feedback controlled for the above mentioned pressure, with a fixed ratio of hydrogen over helium, variable from shot to shot (0 up to $60 \%$ ). The conditioning efficiency was estimated by monitoring partial pressures of hydrogenic molecules using an absolutely calibrated quadrupole mass spectrometer (QMS) remotely connected to a pumping duct located below the Pumped Toroidal Limiter (PTL). A neutral particle analyzer (NPA) provided the isotopic ratio (IR) during reference ohmic discharges. Line integrated $\mathrm{H}_{\alpha}$ radiation measurements were obtained from a central vertical line viewing the PTL.

\section{PLASMA PRODUCTION}

The basic mechanisms of ICRF plasma production for conditioning have been described in [4]. As RF-power is applied to the straps gas breakdown occurs after some delay and shows up in a drop in the antenna RF voltage and in a burst of $\mathrm{H}_{\alpha}$ radiation. Examination of plasma production preceded the assessment of the efficiency of ICWC. When operating the antenna in 0-phasing, a significant increase of coupling efficiency was found (from 15 to $66 \%$ together with considerably smaller breakdown times compared to $\pi$-phasing; $33 \mathrm{~ms}$ instead of $210 \mathrm{~ms}$ for a generator power of $90 \mathrm{~kW}$ and a $\mathrm{H}_{2}$ percentage of $15 \%$ (FIGURE 1). FIGURE 2 shows that the coupling efficiency also improves with higher $\mathrm{H}_{2}$ partial pressures in the vessel. A higher coupling efficiency is important to obtain more reliable antenna operation. The benefits for conditioning are not straightforward due to the complex interplay between plasma and wall. ICWC plasmas are partially ionized so that a higher coupled power leads to a higher plasma density, while the temperature remains about constant $\left(\mathrm{T}_{\mathrm{e}} \approx 3\right.$ eV) [5]. Likely the local conditioning efficiency over the wall area will be altered and eventual higher wall desorption rates will have to compete with higher reionization of desorbed species, which are in turn re-implanted into the walls. The reionization probability in ICWC plasmas is typically higher than $75 \%$ [6]. 
For a constant working pressure the breakdown time and intensity of $\mathrm{H}_{\alpha}$ radiation appears to depend mainly on the applied RF-power; higher powers lead to lower breakdown times and more intense $\mathrm{H}_{\alpha}$ radiation.

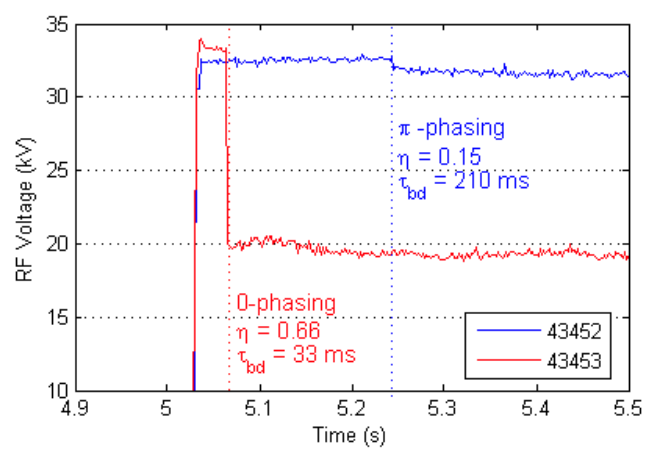

FIGURE 1. Drop in antenna voltage for 2 ICWC discharges resp. in $\pi$ (blue) and 0 phasing (red) indicating plasma breakdown. 0phasing clearly has a shorter breakdown time and better coupling efficiency.

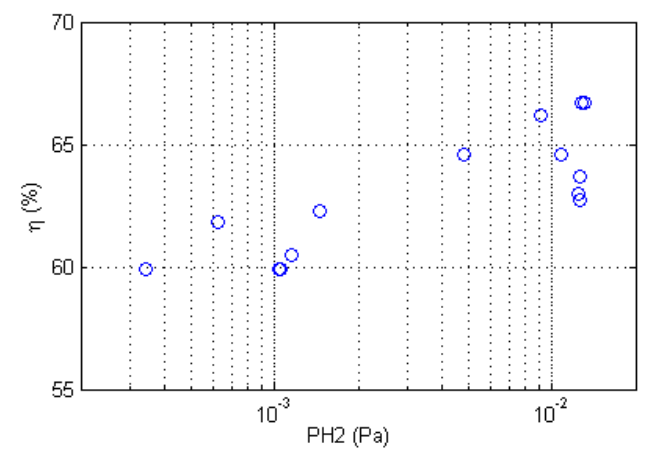

FIGURE 2. Coupling efficiency as a function of the measured $\mathrm{H} 2$ partial pressure in the vessel for subsequent $\mathrm{He} / \mathrm{H}_{2}$ ICWC discharges in 0 -phasing. $\eta$ increases with $\mathrm{P}_{\mathrm{H} 2}$.

After the ICWC experiments shallow arcing traces were found on the surface of the antenna straps indicating the presence of plasma between the straps and the Faraday screen. A preliminary analysis indicates two possible causes. Firstly, in the case of high RF power operation (200 kW at generator) the power was cut several times on a too high reflected power to forwarded power ratio. Secondly, high RF antenna voltages $(\sim 35 \mathrm{kV})$ were measured in the gas breakdown phase for antenna operation in both 0 and $\pi$-phasing (see FIGURE 1). The breakdown phase is known to be a critical phase due to the fast transition from vacuum to plasma conditions [4]. In the case antenna operation in $\pi$-phasing the RF voltage even remained high over the whole discharge duration.

\section{ASSESMENT OF CONDITIONING EFFICIENCY}

The produced ICWC discharge is meant to interact with the wall. The plasma species will impinge on the first wall where they, consequently to numerous interactions with wall particles and following diffusion processes to the surface, lead to desorption of $\mathrm{H}_{2}$, HD and $\mathrm{D}_{2}$ molecules. A part of the impinging species will remain implanted in the wall. A change in isotopic ratio from 4 to $50 \%$ was achieved after only $15 \mathrm{~min}$ of $\mathrm{He} / \mathrm{H}_{2}$ ICWC and 3 min of ICWC in pure Helium, starting from a $\mathrm{D}_{2}$ preloaded wall and measured with NPA during reference ohmic discharges. For every conditioning discharge the total amount of implanted $\mathrm{H}$ and outpumped $\mathrm{D}$ atoms was calculated from gas injection signals and a complete time integration of the $\mathrm{H}_{2}, \mathrm{HD}$ and $\mathrm{D}_{2}$ partial pressures (from the start of gas injection, including discharge duration and post discharge duration). An overview of 4 successive shots is given in Table 1. All these discharges had durations of $60 \mathrm{~s}$ and an RF power of $40 \mathrm{~kW}$ coupled to the 
TABLE 1. Balance of implanted $\mathrm{H}$ and out pumped $\mathrm{D}$ during $\mathrm{He} / \mathrm{H}_{2} \mathrm{ICWC}$ discharges on Tore Supra (TS) with pulse durations of $60 \mathrm{~s}$.

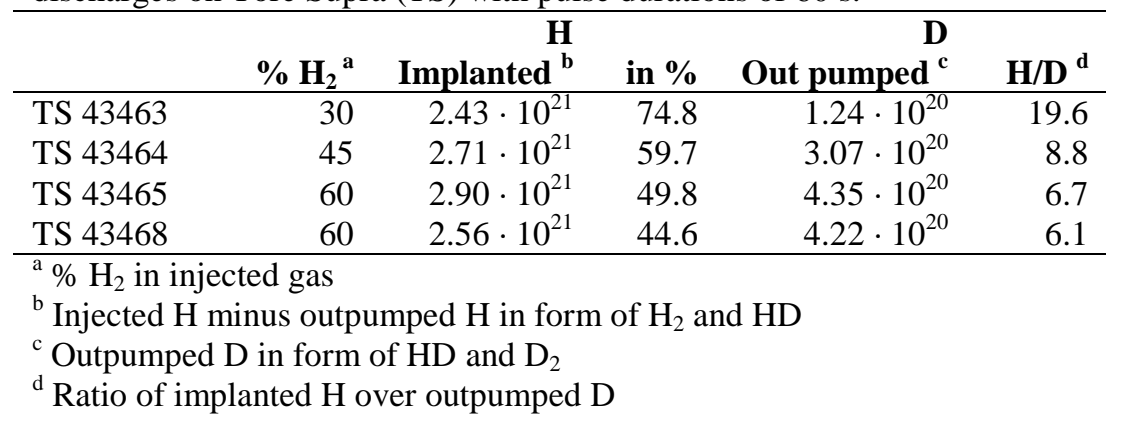

plasma. Two clear effects can be observed. Firstly, the amount of implanted H (b) and outpumped D (c) particles increases with the percentage of $\mathrm{H}_{2}$ in the injected gas (a) whereas the number of $\mathrm{H}$ atoms that one has to implant into the walls for every outpumped D particle decreases with the $\mathrm{H}_{2}$ percentage (d). No discharges in pure $\mathrm{H}_{2}$ have been carried out so far. Secondly, as the wall saturates with $\mathrm{H}_{2}$, less hydrogen is implanted and also less deuterium is pumped out. Using shorter, frequently repeated RF pulses instead of continuous RF operation should reduce re-implantation of walldesorbed species. An optimum has to be sought in order to maximize the ratio between average outpump rates and total implantation. Further results, for example the successful recovery to normal operation after disruptions, are discussed in [7].

\section{CONCLUSION}

$\mathrm{He} / \mathrm{H}_{2}$ ICWC experiments were performed on Tore Supra in order to asses their conditioning and isotope exchange efficiency. It was found that 0-phasing operation of the antenna straps allowed optimal coupling of RF-power to the plasma. A significant change in isotopic ratio in the walls of the vacuum vessel during ICWC was measured, at the price of an important implantation of $\mathrm{H}$ atoms. Analysis of desorption and reimplantation during ICWC discharges indicate the benefit of the use of pulsed discharges.

\section{ACKNOWLEDGMENTS}

This work has been preformed in the frame of collaboration between CEA/Cadarache and ERM/KMS Brussels. The authors gratefully acknowledge the support of the Tore Supra Team.

\section{REFERENCES}

1. J. Roth, et al., Plasma Physics and Controlled Fusion 50, 103001 (20pp) (2008).

2. A0 GDRD 3 01-07-19 R1.0, Design Requirements and Guidelines Level 2 (DRG2), ITER (2001).

3. A. Lyssoivan, et al., this conference

4. A. Lyssoivan, et al., AIP Conference Proceedings 787, 445-448 (2005).

5. E. de la Cal, and E. Gauthier, Plasma Physics and Controlled Fusion 47, 197-218 (2005).

6. E. Gauthier, Journal of Nuclear Materials 241-243, 553 - 558 (1997), ISSN 0022-3115.

7. D. Douai, et al., Proc. of the 36th EPS Conference on Plasma Physics, Sophia (2009) 